Original article

\title{
Hyperglycemia duration impact on anatomical damage level of osteoarthritic articular cartilage in rat models with diabetes mellitus type 1
}

\author{
Ibrahim Njoto
}

University of Wijaya Kusuma Surabaya, Surabaya, Indonesia

Received 26 September 2020, Revised 16 February 2021, Accepted 20 April 2021

(C) 2021, Russian Open Medical Journal

Abstract: Background - Diabetes mellitus caused alteration of chondrocytes morphology of superficial layer on osteoarthritic articular (OA) cartilage in an articular cartilage rat model. These results need to be analyzed in relation to hyperglycemia duration.

Objective - This study evaluates the influence of hyperglycemia on microscopic anatomical damage progression in OA cartilage.

Material and Methods - Thirty-five adult male rats were divided into seven groups: control group, three OA groups, and three OA groups with type 1 diabetes mellitus (DMT-1). For OA groups, the first, second, and third group was sacrificed on the third, fourth, and sixth week respectively after two months maintenance. OA with DMT-1 groups were performed anterior cruciate ligament transaction (ACLT) and were injected streptozotocin intraperitoneally to promote DMT-1 for one-month maintenance. DMT-1.1, DMT-1.2, and DMT-1.3 group was sacrificed on the third, fourth, and sixth week respectively after two months maintenance. The right knee cartilage was taken and processed for histopathology with hematoxylin and eosin staining, then analyzed using a Pritzker scale.

Results - In OA group with DMT-1, hyperglycemia duration $\left(6^{\text {th }}>4^{\text {th }}>3^{\text {th }}\right.$ weeks exposure) increased the level of damage in the OA cartilage compared with the OA group. Pritzker scale observe on deeper abrasiveness of the superficial articular layer, cartilage fissure reaching the middle layer, a more severe decrease in the chondrocytes columnar pattern, changing of matrix integrity, and many sclerotic conditions were provoked by increasing the hyperglycemia duration.

Conclusion - Hyperglycemia duration influenced the damage level in the articular cartilage, increasing the progression of OA disease in animal models.

Keywords: articular, cartilage, chondrocytes, hyperglycemia, sclerotic.

Cite as Njoto I. Hyperglycemia duration impact on anatomical damage level of osteoarthritic articular cartilage in rat models with diabetes mellitus type 1. Russian Open Medical Journal 2021; 10: e0304.

Correspondence to Ibrahim Njoto. Address: Department of Anatomy, Medical Faculty, University of Wijaya Kusuma Surabaya, Dukuh Kupang XXV Street No. 54, Dukuh Kupang, Dukuhpakis, Surabaya 60225, East Java, Indonesia. Phone: +62-31-5686531. Email: ibrahim.njoto@uwks.ac.id.

\section{Introduction}

Hyperglycemia can influence proteins or cellular or molecular glycation of human tissue. The specific characteristics of chondrocytes in the articular cartilage include adaption to low oxygen levels (1\%-7\%) and low sugar levels involving glucose transport (GLUT-1 and GLUT-3) [1, 2]. Chondrocytes live in an isolated microenvironment [3]. Therefore, research on the progressive microscopic anatomical damage on the articular cartilage caused by hyperglycemia duration needs to be conducted.

Anatomy as a basic science has a precious position in the scientific framework, using cellular responses as reasoning bases to understand biomolecular reactions. It is believed that negative influences in the microenvironment of the human tissue as the first impact of molecular or cellular disturbances before such promotes functional distortion, which will be observed as signs or symptoms in a patient. Previous research concluded that hyperglycemia causes the hypertrophy phase of chondrocytes [4]. This condition starts at the superficial layer of the cartilage with a change in the chondrocytes' diagonal diameter and the distances between chondrocytes [5].

The present study analyzed cartilage layers, cartilage fissures, chondrocyte size and distribution, cartilage matrix integrity, and sclerotic condition using the method of Pritzker et al. in a rat model [6]. The results of the present study seek to elucidate whether the osteoarthritic articular (OA) cartilage condition is influenced by hyperglycemia duration. The anterior cruciate ligament transaction (ACLT) was used to create the osteoarthritic condition [7]. The present study used ACLT maintenance as representative of posttraumatic OA (PTOA), which comprises $12 \%$ of the total OA cases. A previous study showed that $50 \%$ of knee trauma cases lead to OA after five to 10 years, which will increase to $75 \%$ when followed by an intra-articular fracture [8]. Therefore, the present study analyzed microscopic anatomical changes in an OA cartilage rat model to understand the evolution of the osteoarthritic condition in accordance with varying hyperglycemia durations representative of type 1 diabetes mellitus (DMT- 1 ). This study used the occurrence of hyperglycemia in OA as a prevention base and reduced $\mathrm{OA}$ disease progression and morbidity by 
revealing the impact of the level of damage between the $\mathrm{OA}$ cartilage group and the OA cartilage with DMT-1 group.

\section{Material and Methods}

\section{Animal subjects}

This study received approval from the research ethics committee of Brawijaya University, Malang, East Java, Indonesia No. 882-KEP-UB. Thirty-five adult male rats (Rattus norvegicus strain Wistar) aged five to six months old and weighing 300 to $400 \mathrm{~g}$ were included. The animal models were maintained in the Animal Hotel at the Laboratory of Biosain Institute, Brawijaya University, Malang, Indonesia. Environmental conditions are maintained at a temperature of $22^{\circ} \mathrm{C}-23^{\circ} \mathrm{C}$, humidity at $76 \%-80 \%$, and a $12 \mathrm{~h}$ light cycle (lights turn on at $06.00 \mathrm{AM}$ and turn off at 06.00 PM). All subject animals were housed in $370 \times 290 \times 120 \mathrm{~mm}$ cages. They were fed standard Japfa Comfeed concentrate (content: $12 \%$ of water, $16 \%-18 \%$ of crude protein, $3 \%-7 \%$ of crude fat, $8 \%$ of maximum crude fiber, $10 \%$ of ash, $0.8 \%-1.0 \%$ of calcium, and $0.6 \%-0.8 \%$ of phosphorus) and ad libitum water. During maintenance, the animal health was monitored twice a day at feeding times in the mornings and evenings.

\section{Experimental design}

This type of research is a purely laboratory experimental study, involving the subject of the Wistar mouse model (Rattus norvegicus). All subjects were randomly divided among seven groups with five rats included in each group as follows: a control group $(\mathrm{N})$, three groups treated with ACLT to create OA condition (ACLT 1, 2, and 3), and three groups treated with ACLT followed by $15 \mathrm{mg} / \mathrm{kg}$ body weight intraperitoneal streptozotocin injection for three days to produce the hyperglycemia condition (DMT-1.1, -1.2, and -1.3). All subjects were given $30 \mathrm{~g}$ of standard diet twice a day in the morning and afternoon. The ACLT groups were maintained for two months after treatment. After that, the groups were sacrificed as follows: three (ACLT 1), four (ACLT 2), and six (ACLT 3) weeks after two months of treatment. The DMT-1 groups were maintained for one month after ACLT treatment and then given the streptozotocin intraperitoneal injection. After that, they were maintained for another month. Similar to ACLT group, the DMT$1.1,-1.2$, and -1.3 groups were sacrificed on the third, fourth, and sixth weeks, respectively, after two months of maintenance. All animal models were sacrificed by physical euthanasia (cervical dislocation) under anesthesia (intraperitoneal injection of
$80 \mathrm{mg} / \mathrm{kg}$ body weight Ketamine and $10 \mathrm{mg} / \mathrm{kg}$ body weight Xylazine). The present study used a duration-of-time experimental design, which considered the right knees of the rats with $O A$ without hyperglycemia versus with hyperglycemia.

\section{Histopathological analysis}

The right knee joint was flooded in RapidCal ${ }^{\mathrm{TM}}$ Immuno as a decalcifying agent to make joint and bone softer and easier to cut by microtome. The tissue was then proceeded to produce thin slices of cartilage tissue in paraffin block. The slices of cartilage tissue were laid at object glass, then performed the hematoxylin and eosin staining, and scored the articular damage level according to the Pritzker scale [6].

\section{Statistical analysis}

We used one-way Analysis of Variance and Pearson Correlation by SPSS Program $22^{\text {th }}$ version to analyze the research data (Table 1). The blood glucose of OA-like group and OA-like with DMT-1 was analyzed using a correlation factor to anatomical damage level of OA articular cartilage by Pritzker Scale; using the Pearson's correlation yielding correlation coefficients $(\pi)$ and $p$ values at the times of hyperglycemia duration: the $3^{\text {rd }}$ week; the $4^{\text {th }}$ week and the $6^{\text {th }}$ week. The results are presented in tables and figures.

\section{Results}

The results of the statistical analysis show that the correlation coefficient $(r)$ of the OA + DMT-1 group rose from 0.480 on the third week to 0.541 on the fourth week and to 0.562 on the sixth week. This means that the hypothesis, hyperglycemia duration correlated to an increase in the Pritzker scale cartilage damage level.

Table 1. The statistical analysis of Pearson Correlation: Hyperglycemia duration correlated with the Pritzker scale

\begin{tabular}{|c|c|c|c|c|c|c|c|}
\hline \multirow{3}{*}{ Group } & & \multicolumn{6}{|c|}{ Duration } \\
\hline & & \multicolumn{2}{|c|}{$3^{\text {rd }}$ Week } & \multicolumn{2}{|c|}{$4^{\text {th }}$ Week } & \multicolumn{2}{|c|}{$6^{\text {th }}$ Week } \\
\hline & & $r$ & $\mathrm{p}$ & $r$ & $\mathrm{p}$ & $r$ & $p$ \\
\hline $\mathrm{OA}$ & $\begin{array}{l}\text { Hyperglycemia } \\
\text { Pritzker Scale }\end{array}$ & 0.649 & 0.042 & 0.633 & 0.049 & 0.707 & 0.022 \\
\hline $\mathrm{OA}+\mathrm{DMT}-1$ & $\begin{array}{l}\text { Hyperglycemia } \\
\text { Pritzker Scale }\end{array}$ & 0.480 & 0.026 & 0.541 & 0.015 & 0.562 & 0.023 \\
\hline
\end{tabular}

Table 2. Pritzker scale description as a guide for the parameters of articular cartilage damage level. This scale measures the area of the cartilage layer, cartilage fissure, chondrocytes size and distribution, matrix integrity, and sclerotic formation

\begin{tabular}{|c|c|c|c|c|c|c|}
\hline Level & Status & Cartilage layer & Cartilage fissure & $\begin{array}{c}\text { Chondrocytes Size \& } \\
\text { distribution }\end{array}$ & Matrix integrity & $\begin{array}{c}\text { Sclerotic } \\
\text { formation }\end{array}$ \\
\hline 1 & Surface intact & Surface intact & - & Appropriate orientation & $\begin{array}{c}\text { Normal architecture } \\
\text { (homogeny) }\end{array}$ & $(-)$ \\
\hline 2 & $\begin{array}{l}\text { Surface } \\
\text { discontinuity }\end{array}$ & $\begin{array}{l}\text { Discontinuity at superficial } \\
\text { zone }\end{array}$ & Deep fibrillation & $\begin{array}{l}\text { Disorientation of chondrocyte } \\
\text { columns; cell death }(+) ; \\
\text { proliferation (clusters); } \\
\text { hypertrophy }\end{array}$ & $\begin{array}{l}\text { Depletion matrix } 1 / 3 \text { upper } \\
\text { cartilage zone }\end{array}$ & $(-)$ \\
\hline 3 & $\begin{array}{l}\text { Vertical } \\
\text { fissures (clefts) }\end{array}$ & $\begin{array}{l}\text { As above; Cartilage domains } \\
\text { adjacent to fissures }\end{array}$ & $\begin{array}{c}\text { As above; }(+) \text { To mid zone; branched } \\
\text { fissures }\end{array}$ & $\begin{array}{l}\text { Cell death (+); regeneration } \\
\text { (clusters); hypertrophy }\end{array}$ & $\begin{array}{l}\text { Depletion matrix } 2 / 3 \text { upper } \\
\text { cartilage zone; New collagen } \\
\text { formation (heterogeny) }\end{array}$ & $(-)$ \\
\hline 4 & Erosion & $\begin{array}{l}\text { Delamination of superficial } \\
\text { layer; mid layer cyst formation }\end{array}$ & $\begin{array}{c}\text { As above; }(+) \text { To mid zone cartilage } \\
\text { loss }\end{array}$ & $\begin{array}{l}\text { Cell death }(++) \\
\text { low distribution }\end{array}$ & Cartilage matrix loss & $(+)$ \\
\hline 5 & Denudation & $\begin{array}{c}\text { Sclerotic bone or fibrocartilage } \\
\text { repair }\end{array}$ & To bone surface & $\begin{array}{l}\text { Decreasing distribution, } \\
\text { hypertrophy }\end{array}$ & Cartilage matrix loss & $(+)$ \\
\hline 6 & Deformation & Bone remodeling & $\begin{array}{l}\text { Microfracture with fibrocartilaginous } \\
\text { and osseous repair }\end{array}$ & Cell death $(+++)$ calcification & Ossification of matrix & $\begin{array}{l}\text { Osteophyte } \\
\text { formation }\end{array}$ \\
\hline
\end{tabular}




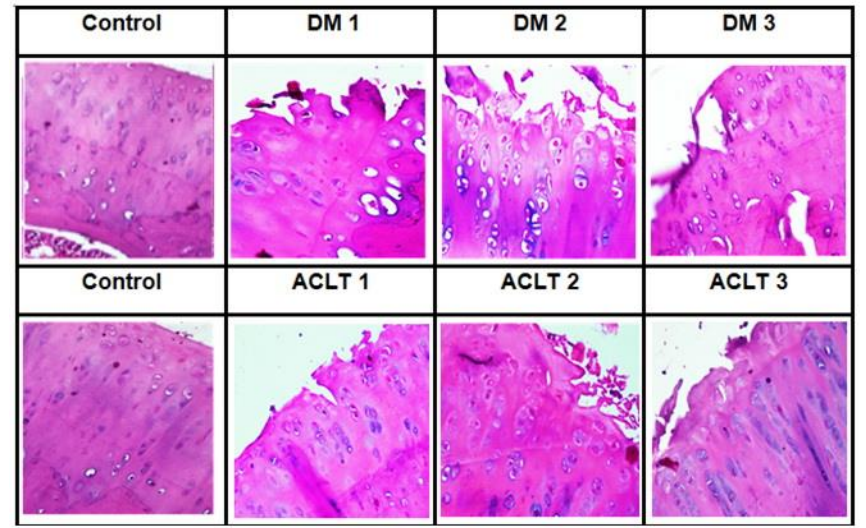

Figure 1. Comparison of the microscopic anatomy of the articular cartilage formation and damage evaluation with H\&E (light microscope 40x) of the DMT-1 groups and the ACLT groups without duration of hyperglycemia. It showed that all groups had severe cartilage superficial layer damage, with it being more severe in the DMT-1 groups than in the ACLT groups.

Table 3. Analysis on the microscopic anatomical damage levels in the articular cartilage by Pritzker scale of the $\mathbf{N}$ group, three DMT-1 groups and three ACLT groups

\begin{tabular}{lccccccc}
\hline \multirow{5}{*}{ N group } & $\begin{array}{c}\text { DM 1 } \\
\text { group }\end{array}$ & $\begin{array}{c}\text { DM 2 } \\
\text { group }\end{array}$ & $\begin{array}{c}\text { DM 3 } \\
\text { group }\end{array}$ & $\begin{array}{c}\text { ACLT 1 } \\
\text { group }\end{array}$ & $\begin{array}{c}\text { ACLT 2 } \\
\text { group }\end{array}$ & $\begin{array}{c}\text { ACLT 3 } \\
\text { group }\end{array}$ \\
\hline \multirow{5}{*}{ Pritzker scale } & 1) 2 & 1) 1 & 1) 1 & 1) 2 & 1) 0 & 1) 1 & 1) 0 \\
& 2) 0 & 2) 2 & 2) 4 & 2) 4 & 2) 2 & 2) 2 & 2) 1 \\
Mean 0 & 3) 3 & 3) 3 & 3) 4 & 3) 2 & 3) 1 & 3) 3 \\
Std. Deviation & 4) 0 & 4) 2 & 4) 1 & 4) 1 & 4) 2 & 4) 1 & 4) 1 \\
& 5) 0 & 5) 3 & 5) 3 & 5) 4 & 5) 1 & 5) 3 & 5) 0 \\
& 0.4 & 2.2 & 2.4 & 3 & 1.4 & 1.6 & 1.0 \\
\end{tabular}

The OA cartilage damage level was analyzed using the Pritzker scale (Table 2) by observing articular cartilage components such as the superficial and all layers of cartilage, cartilage fissures, chondrocyte size and distribution, matrix integrity, and sclerotic formation.

Considering the results of histopathological analysis, Figure 1 shows that fissures in the superficial cartilage layers of the DMT-1 groups were more severe than in the ACLT groups. Chondrocyte size and distribution in the DMT-1 groups demonstrated a reduction in the columnar pattern. The matrix integrity of the cartilage displayed more apoptotic bodies and sclerotic formation in the DMT-1 groups than in the ACLT groups.

As shown in Table 2, the Pritzker scale of the DMT-1 groups increased during hyperglycemia duration, which shows an increase in the severity of the damage in the articular cartilage; whereas, the Pritzker scale of the ACLT groups decreased during the maintenance period without hyperglycemia duration, which shows a decrease in the severity of the damage in the articular cartilage or a regenerating response (Table 3 ).

Based on the Pritzker scale (Table 3), the results of the data analysis of the articular damage level showed that the DMT-1 groups experienced increasing damage severity, ranging from a mean of 2.2 in the DMT-1.1 group, 2.4 in the DMT-1.2 group, and 3 in the DMT-1.3 group. Outcomes showed that, at the microscopic anatomical level, more and more damage was happening in the DMT-1 groups with longer hyperglycemia durations. Table 2 shows the results of the DMT-1 groups. The DMT-1.1 group presented a score from levels 1 to 3 , where the cartilage layer was abrasive from the superficial to early mid zone and had branching fissures, chondrocyte death, missing chondrocyte column formation and regeneration form, chondrocytes in the hypertrophy phase, decreased matrix integrity by a depletion in the matrix of one-third to two-thirds of the upper layer of the cartilage, and less sclerotic formation. This condition lasted an extra week in the DMT-1.2 group, which showed various score results for levels 1 to 4 , with more progressive damage such as deeper cartilage fissures and branching to the mid zone, chondrocyte death, less regeneration, hypertrophic chondrocytes, matrix loss, and sclerotic formation. The score of the DMT-1.2 group was worse than in the DMT-1.1 group. When hyperglycemia duration was continued for two more weeks, as in the DMT-1.3 group, the level 4 score was dominant in five rats. This means that cartilage damage progression was increased and showed no regeneration cluster formation. The progression of the articular cartilage damage level was increased by the impact of hyperglycemia duration.

Furthermore, the results of the ACLT groups revealed that the articular damage level on the Pritzker scale (Table 3) decreased from a mean of 1.6 in ACLT 2 to 1.4 in ACLT 1 and 1.0 in the ACLT 3 group at sacrifice. Further, the Pritzker scale revealed a decrease in the mean of severity upon comparison with the ACLT 2 group (mean: 1.6), ACLT 1 group (mean: 1.4), and ACLT 3 group (mean: 1.0). These showed that the ACLT 2 group, after 12 weeks of maintenance, had the highest level on the Pritzker scale, varying from 1 to 3, whereas after two more weeks, the ACLT 3 group showed a reduction in Pritzker scale level to 1 in five rats, and to 0 at samples No.1 and 5 (these conditions showed the response of cartilage regeneration by chondrocytes over time). When this condition continued for two more weeks, after 14 weeks of maintenance, the domination of level 1 on the Pritzker scale was noted. These impressive results in the ACLT groups revealed a reduction in the progression of articular damage by increasing chondrocyte cellular responses such as chondrocyte proliferation and the prehypertrophic phase, chondrocyte column formation, matrix integrity, and fissure depth reduction.

\section{Discussion}

The resulting data on the analyzed articular damage level using the Pritzker scale revealed that the DMT-1 groups experienced increasing damage severity. The analysis exposed at the microscopic anatomical level that progressively greater damage was happening in the DMT-1 groups under hyperglycemia. Hyperglycemia directly acts inside the chondrocytes on the cell membrane because of GLUT-1 and GLUT-3 [2]. It changes the chondrocytes' intracellular conditions, disturbing the glycolytic level and leading to age-related hypertrophic phase [9]. Our results also showed that the influence of time/the hyperglycemia duration seriously impacted the severity of the OA disease by increasing its progression.

Furthermore, without the influence of hyperglycemia duration, the articular damage level in the ACLT groups declined. These results supported the regenerative response of chondrocytes in the articular cartilage that received PTOA treatment without the influence of hyperglycemia.

The present study used a rat OA model treated with ACLT maintenance to externally induce OA over four to six weeks [10]. Hyperglycemia causes glycation of molecular proteins or cellular 
proteins in all human tissues, including the articular cartilage [11]. When hyperglycemia reaches the articular cartilage, it will be considered as a side effect because the articular cartilage only has chondrocytes as resident cells [12]. It will cause some change or modification of molecular components or proteins located in the cartilage matrix, affecting their original function. Chondrocytes usually live in a microenvironment with a lower glucose level than the blood glucose level, and it had glucose transport noninsulin dependence (GLUT-1 and GLUT-3) $[2,13]$.

Therefore, when hyperglycemia reached the matrix, continually affecting the intracellular area of chondrocytes, it caused something else. A previous study of the results of diabetes in OA suggested an increase in the severity of this disease [14]. In addition, another previous study found that the specific condition of articular change affected avascular, aneural and lymphatic tissue, so the major chondrocyte response was as of a residential cartilage cell [15]. It is believed that molecules or proteins change, causing alteration in the original function, influencing chondrocytes to receive compression at the superficial layer first, followed by a more in-depth component of articular cartilage [16]. The present study showed opposite results between the DMT-1 and ACLT groups, supporting that hyperglycemia duration had different impacts on the OA articular cartilage of rats over time.

By analyzing a superficial layer of the articular cartilage, the results of the DMT-1 groups revealed that DMT-1 increased the severity of OA disease [5], which had a more in-depth and branched fissure. This condition was caused by reducing the matrix integrity due to changing the chondrocyte phase from proliferating and hypertrophic phases to the hypertrophic phase; therefore, the chondrocyte size was increased in the DMT-1 groups, especially in the DMT-1.3 group, which had the longest duration. Hypertrophic chondrocytes would produce more collagen type $\mathrm{X}$ and catabolic factors such as proinflammatory cytokines [17], so it would accelerate articular cartilage matrix degradation. The hypertrophic phase also accelerated chondrocyte apoptosis or cell death [18]. Therefore, progressively greater chondrocyte expiry was observed in the DMT-1 groups, especially in the DMT-1.3 group, which involved the most extended hyperglycemia duration. The changing of collagen production by chondrocytes in the hypertrophic phase from collagen type II to type $X$ was caused by the heterogeneity of matrix colorization in hematoxylin and eosin staining. We observed the heterogeneous color of the matrix in a microscopic anatomical sample, which was a sign of the changing matrix integrity and reduced matrix elasticity. In addition, many sclerotic conditions were found in the articular cartilage matrix in the DMT1.3 group. Furthermore, reduced chondrocyte column formation means that hyperglycemia duration was limiting the chondrocyte regeneration response.

The glycation process impacts the intracellular component of chondrocytes the same way molecular proteins of the aortic endothelial cell are modified [19]. All above characteristics of the microscopic anatomical level were analyzed as cellular and tissue changes caused by hyperglycemia duration with an increasing value on the Pritzker scale, which means increased progression in the damage of the articular cartilage. The present study proposes that if hyperglycemia duration maintenance is extended for some extra weeks, it will continue to increase the progression in the damage level of the articular cartilage because the glycation process continues to be impacted severely with molecular and cellular component changes.
The resulting data of the ACLT groups showed that OA without the influence of hyperglycemia duration exhibited a decreasing level on the Pritzker scale. It means slowing the progression of OA cartilage damage, reducing the deepness of the cartilage fissure, increasing chondrocyte quantity around the fissure and column formation, increased chondrocyte phases, reducing apoptotic bodies, and a more homogenous colorization in the cartilage matrix. All above characteristics in the ACLT groups were in contrast to the microscopic anatomical results in the DMT-1 groups because a regenerative response was seen in the ACLT groups without the influence of hyperglycemia duration. The present study results showed that hyperglycemia duration needs to be controlled as soon as possible to prevent the progression of OA disease.

\section{Conclusion}

This study showed that hyperglycemia duration caused increasing progressiveness of OA cartilage damage due to an increase in deeper articular cartilage fissures in the superficial layer, a change to the hypertrophic phase in chondrocytes, augmented chondrocyte expiry, reduced matrix integrity, and sclerotic formation, as measured by the Pritzker scale. Therefore, the damage level in the OA cartilage in the rat model becomes severe because of DMT-1. Hyperglycemia badly influences articular matrix glycation and chondrocyte response to matrix regeneration. Therefore, hyperglycemia should be avoided as soon as possible to reduce severity and progression of OA disease.

\section{Acknowledgments}

The author would like to thank the Ministry of Research and Technology/National Research and Innovation Agency of the Republic of Indonesia for the research funding and Medical Faculty, University of Wijaya Kusuma Surabaya for facilitating this study.

\section{Conflict of interest}

The authors declare that he has no conflict of interest.

\section{Ethical approval}

All procedures performed in studies involving animals were in accordance with the ethical standards of the institution or practice at which the studies were conducted.

\section{References}

1. Wilkins RJ, Browning JA, Ellory JC. Surviving in a matrix: Membrane transport in articular chondrocytes. J Membr Biol 2000; 177(2): 95-108. https://doi.org/10.1007/s002320001103.

2. Mobasheri A, Richardson S, Mobasheri R, Shakibaei M, Hoyland JA. Hypoxia inducible factor-1 and facilitative glucose transporters GLUT1 and GLUT3: Putative molecular components of the oxygen and glucose sensing apparatus in articular chondrocytes. Histol Histopathol 2005 . 20(4): 1327-1238. https://doi.org/10.14670/hh-20.1327.

3. Loeser RF. Aging and osteoarthritis: The role of chondrocyte senescence and aging changes in the cartilage matrix. Osteoarthritis Cartilage 2009; 17(8): 971-979. https://doi.org/10.1016/j.joca.2009.03.002.

4. Salinas D, Minor CA, Carlson RP, McCutchen CN, Mumey BM, June RK. Combining targeted metabolomic data with a model of glucose metabolism: Toward progress in chondrocyte mechanotransduction. PLOS One 2007; 12(1): 0168326. https://doi.org/10.1371/journal.pone.0168326. 
5. Njoto I, Soekanto A, Ernawati E, Abdurrachman A, Kalim H, Handono K, et al. Chondrocyte intracellular matrix strain fields of articular cartilage surface in hyperglycemia model of rat: Cellular morphological study. Med Arch 2018; 32(5): 348-351. https://doi.org/10.5455/medarh.2018.72.348-351.

6. Pritzker KPH, Gay S, Jimenez SA, Ostergaard K, Pelletier J-P, Revell PA, et al. Osteoarthritis cartilage histopathology: Grading and staging. Osteoarthr Cartil 2006; 14(1): 13-29. https://doi.org/10.1016/i.joca.2005.07.014.

7. Kraeutler MJ, Wolsky RM, Vidal AF, Bravman JT. Anatomy and biomechanics of the native and reconstructed anterior cruciate ligament: Surgical implications. J Bone Joint Surg Am 2017; 99(5): 438445. https://doi.org/10.2106/jbjs.16.00754.

8. Chubinskaya S, Haudenschild D, Gasser S, Stannard J, Krettek C, Borrelli $\mathrm{J} \mathrm{Jr}$. Articular cartilage injury and potential remedies. J Orthop Trauma 2015; $29 \quad$ Suppl 12 (Suppl 12): S47-S52. https://doi.org/10.1097/bot.0000000000000462.

9. Chubinskaya S, Kumar B, Merrihew C, Heretis K, Rueger DC, Kuettner $\mathrm{KE}$. Age-related changes in cartilage endogenous osteo- genic protein1 (OP-1). Biochim Biophys Acta 2002; 1588(2): 126-134. https://doi.org/10.1016/s0925-4439(02)00158-8.

10. Glasson SS, Blanchet TJ, Morris EA. The surgical destabilization of the medial meniscus (DMM) model of osteoarthritis in the 129/SvEv mouse. Osteoarthritis Cartilage 2007; 15(9): 1061-1069. https://doi.org/10.1016/i.joca.2007.03.006.

11. Lohmander LS, Englund PM, Dahl LL, Roos EM. The long-term consequence of anterior cruciate ligament and meniscus injuries: Osteoarthritis. Am J Sports Med 2007; 35(10): 1756-1769. https://doi.org/10.1177/0363546507307396.

12. Kwon S, Kim YJ, Kim MK. Effect of fructose or sucrose feeding with different levels on oral glucose tolerance test in normal and type 2 diabetic rats. Nutr Res Pract 2008; 2(4): 252-258. https://doi.org/10.4162/nrp.2008.2.4.252.

13. Schett G, Kleyer A, Perricone C, Sahinbegovic E, lagnocco A, Zwerina J, et al. Diabetes is an independent predictor for severe ostheoarthritis: Results from a longitudinal cohor study. Diabetes Care 2013; 36(2): 403-409. https://doi.org/10.2337/dc12-0924.

14. Clouet J, Vinatier C, Merceron C, Pot-vaucel M, Maugars Y, Weiss P, et al. From osteoarthritis treatments to future regenerative therapies for cartilage. Drug Discov Today 2009; 14(19-20): 913-925. https://doi.org/10.1016/j.drudis.2009.07.012.

15. Baugé $C$, Duval E, Ollitrault $D$, Girard N, Leclercq $S$, Galéra $P$, et al. Type II TGF $\beta$ receptor modulates chondrocyte phenotype. Age (Dordr) 2013; 35(4): 1105-1116. https://doi.org/10.1007/s11357-012-9433-7.

16. Sakai N, Hashimoto C, Yarimitsu S, Sawae Y, Komori M, Murakami T. A functional effect of the superficial mechanical properties of articular cartilage as a load bearing system in a sliding condition. Biosurf Biotribol 2016; 2(1): 26-39. https://doi.org/10.1016/i.bsbt.2016.02.004

17. Behonick DJ, Werb Z. A bit of give and take: The relationship between the extracellular matrix and the developing chondrocyte. Mech Dev 2003; 120(11): 1327-1336. https://doi.org/10.1016/j.mod.2003.05.002.

18. Shane Anderson A, Loeser RF. Why is osteoarthritis an age-related disease? Best Pract Res Clin Rheumatol 2010; 24(1): 15-26. https://doi.org/10.1016/j.berh.2009.08.006.

19. VogI-Willis CA, Edwards IJ. High-glucose-induced structural changes in the heparan sulphate proteoglycan, perlecan, of cultured human aortic endothelial cells. Biochim Biophys Acta 2004; 1672(1): 36-45. https://doi.org/10.1016/j.bbagen.2004.02.005.

Authors:

Ibrahim Njoto - Dr, M.Hum, Assistant Professor of Anatomy at University of Wijaya Kusuma Surabaya, Surabaya, East Java, Indonesia. https://orcid.org/0000-0001-5358-1695. 